\title{
ANALYSIS OF HEAVY METAL COMPONENTS IN SOME URBAN PONDS IN RAJSHAHI, BANGLADESH
}

\author{
Fawzia Adib Flowra*, Jibon Kumar Ghosh, Md. Abu Sayed Jewel, \\ Anannya Sen Tumpa and M. Afzal Hussain
}

Department of Fisheries, University of Rajshahi, Rjashahi-6205, Bangladesh

*Corresponding Author. mail: flowrabd@yahoo.com

\begin{abstract}
An experiment was conducted to determine the heavy metal pollution status of selected six ponds in Rajshahi City Corporation for a period of 7 months from June, 2008 to December, 2008. Among heavy metals the mean values of $\mathrm{Cu}, \mathrm{Mn}, \mathrm{Zn}, \mathrm{Fe}$ and $\mathrm{Pb}$ varied from $0.05 \pm 0.02$ (Pond-5) to $1.79 \pm 0.88 \mathrm{mg} / \mathrm{l}$ (Pond-4), $21.30 \pm 2.64$ (Pond-6) to 76.57 $\pm 30.90 \mathrm{mg} / \mathrm{l}$ (Pond-1), $0.08 \pm 0.02$ (Pond-6) to $1.25 \pm 0.79 \mathrm{mg} / \mathrm{l}$ (Pond-4), $0.75 \pm 0.10$ (Pond3) to $1.87 \pm 0.53 \mathrm{mg} / \mathrm{l}$ (Pond-5) and $0.14 \pm 0.12$ (Pond-6) to $4.92 \pm 1.66 \mathrm{mg} / \mathrm{l}$ (Pond-4) respectively. This study indicated that the Pond-2 covered by garbage, polythene and other wastes, Pond-3 and Pond-4 received industrial wastes were moderately polluted whereas the Pond-1 received City corporation wastes was less polluted.
\end{abstract}

Keywords: Heavy metal, pollution, urban ponds, Bangladesh

সারাংশ: রাজশাহী সিটি কর্পোরেশনের নির্ধারিত ছয়টি পুকুরে ভারী ধাতব দূষণের অবস্থা নির্ধারণ করার জন্য জুন ২০০b- থেকে ডিসেম্বর ২০০b- পর্যন্ত ৭ মাস ব্যাপি একটি

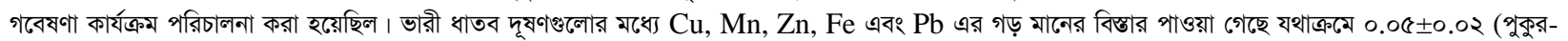

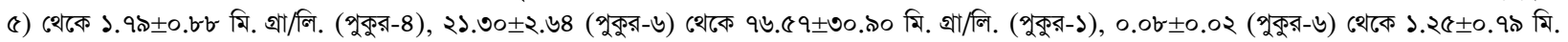

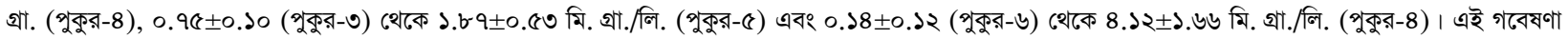
থেকে জানা যায় যে, পুকুর-২ আবর্জনা, পলিথিন এবং অন্যান্য বর্জ্য, পুকুর-৩ এবং পুকুর-৪ এ শিল্পকারখানার বর্জ্য দ্বারা মোটামুটিভাবে দূষিত যেখানে পুকুর-১ সিটি কর্পোরেশন বর্জ্য দ্বারা তুলনামূলকভাবে কম দূষিত।

\section{Introduction}

Water pollution is a global problem, affecting both the industrialized and the developing nations. The water pollution problems in the nation, however, are quite different in many respects. Heat, toxic metals, acids, sediment, animal and human wastes, and synthetic organic compounds foul the waterways of developed nations. Human and animal wastes, sediment and pathogenic organisms head the list in the non-industrialized nations. All kinds of wastes, both solid and liquid forms are dumped indiscriminately into open waters resulting in pollution of the aquatic environment (IUCN 1991). The term 'water pollution' is referred to the addition to water of an excess of material (or heat) that is harmful to humans, animals, to desirable aquatic life or otherwise causes significant departures from the normal activities of various living communities in or near bodies of water (Trivedi and Raj 1997). Different types of water bodies get polluted due to the discharge of effluents from the industries, domestic waste, land and agricultural drainage. This results in the degradation of water quality of the water resources. Water pollution is greatly affected for the presence of heavy metal. Among the various means of pollutions of pond are washing of the clothes, cleaning of animals, dumping of the waste by unauthorized small scale units functioning in the surrounding to the pond. Haslam (1991) reported that due to the great expansion in the urbanization and industrialization, most of the water bodies especially developed countries are polluted. According to Bush et al. (1988), main sources of heavy metals in water due to municipal wastes, industrial wastes and infiltration waters from the top soil layers. Pollution due to industrial waste disposal has not come up as a major problem since our country is industrially less developed. Whatever industries we have (fertilizer, sugar, cement, refinery, electroplating units, plastic, textile etc.) are hardly equipped with any pollution control measure. The release of waste effluents into aquatic system have given rise to heavy localized contamination hazards and threatens the environment as a pollutant.

It is known by all that ponds are very important water bodies in the country. According to World Bank (1991) Bangladesh ranks at the top for production of fresh water fishes $(4076 \mathrm{~kg} / \mathrm{sq} . \mathrm{km}$.) and in terms of total production and per capita fish production of about 5.5 $\mathrm{kg}$, it stands third after China and India. The total pond fisheries production was $1219736 \mathrm{mt}$ (DoF, 2012) whereas in Rajshahi district the total fish production was $21764 \mathrm{mt}$ (DoF 2008). The roles of these ponds are very functional for freshwater aquaculture. Most of the research works have been focused on the analysis of water quality like physic-chemical or biological parameters. But very little works have been done to generate data on the others components like heavy metal in fish ponds. Some related works in this point were done by Jop (1980), Manga (1983), Gaigher and Krause (1989), Tariq et al. (1996), Ehshan et al. (1997), Kabir et al. (1997), Hossain (1998), Saha and Hossain (2002) and Javed (2003). So, it is necessary to assess water pollution status and its impact on fisheries resources of pond considering the adverse effects of municipal sewage, industrial effluents on water quality. 
The present study has been aimed to conduct the determination of heavy metal components in selected urban fish ponds in Rajshahi.

\section{Materials and Methods}

Water samples were collected once in a month at 10 a.m.11 a.m. for a period of seven months from June, 2008 to December, 2008 in six fishponds which were situated at Hadirmore (1), Sonadighir more (2), BSCIC (Bangladesh Small and Cottage Industries Corporation) area $(3,4)$ and Kazihata $(5,6)$ of Rajshahi City Corporation. Collected samples were analyzed by the Institute of environmental sciences, University of Rajshahi. The heavy metal $(\mathrm{Cu}, \mathrm{Pb}$, $\mathrm{Zn}, \mathrm{Fe}, \mathrm{Mn}$ ) concentration of water samples was determined by using Spectrophotometer (Model Shimadzu UV1601 PC, Made in Japan).

\section{Results and Discussion}

The data of heavy metal components on some ponds were recorded during the study period are presented below:

Copper (Cu): Copper concentration ranged between $0.01 \mathrm{mg} / \mathrm{l}$ to $3.52 \mathrm{mg} / \mathrm{l}$ in water samples Fig -1 but high concentration these elements in the water are toxic. Increased copper concentration in aquatic environment resulting from industrial development was favour copper uptake by aquatic animals including fish (Takasusuki et al. 2004). The highest values of Cu were recorded in the month of September at pond- 4 and the lowest value was found in the month of December at pond-5. The mean value of copper was varied from $0.05 \pm 0.02$ (Pond-5) to $1.79 \pm 0.88 \mathrm{mg} / \mathrm{l}$ (Pond-4). This finding clearly indicated water pollution due to industrial effluents and also agreed with the findings of Moore and Ramamoorthy (1984) and Meade (1989). Abel and Green (1981) reported that different wastes of industries and textiles cause serious pollution due to the presence of the heavy metal salts of copper.

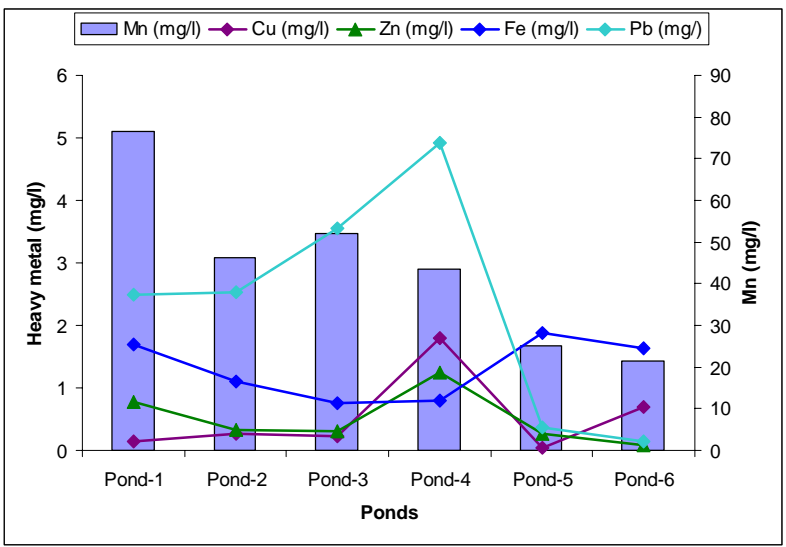

Figure 1 Mean variation in heavy metals
Manganese (Mn): The mean value of manganese was varied from $21.30 \pm 2.64$ (Pond-6) to $76.57 \pm 30.90 \mathrm{mg} / \mathrm{l}$ (Pond-1) Fig-1. Significant concentration of manganese was found almost in the same range throughout the study period. According to Nazneen (1980), pond waters are known to contain relatively large quantities of manganese usually in the range of $500-5000 \mathrm{mg} / \mathrm{m}^{3}$. Harvey (1949) has suggested that manganese content controls the productivity but affects more likely those factors which controlplant and animal growth.

According to the report of WHO (1984), the drinking water should contain only $0.1 \mathrm{mg} / \mathrm{l}$ manganese while the observed values for streams and rivers are between $0.01-2200 \mathrm{mg} / \mathrm{l}$ as reported by McCutcheon et al. (1992). Javed (1999) reported high concentration of manganese causes metallic ion pollution.

Zinc (Zn): Zinc concentration varied from $0.05 \mathrm{mg} / \mathrm{l}$ to $5.19 \mathrm{mg} / \mathrm{l}$ in water samples Fig -1 but this element is toxic to fish and other aquatic organisms as well as human beings (Kupchella and Hyland 1993). The highest values of $\mathrm{Zn}$ were recorded in the month of September at pond- 4 and the lowest value was found in the month of December at pond- 6 . The mean value of toxic ammonia was varied from $0.08 \pm 0.02$ (Pond-6) to $1.25 \pm 0.79 \mathrm{mg} / \mathrm{l}$ (Pond-4). This finding clearly indicated water pollution due to municipal wastes, industrial effluents etc. and also agreed with the findings of Moore and Ramamoorthy (1984) and Meade (1989).

Iron (Fe): An irregular pattern of variations was observed in the distribution of total iron. The present data shows more fluctuations throughout the water of pond with a slight increase in the iron level. The mean value of iron was varied from $0.75 \pm 0.10$ (Pond-3) to $1.87 \pm 0.53 \mathrm{mg} / \mathrm{l}$ (Pond-5) Fig -1 . Iron also plays a very important role and it is evidently an oxygen carrying substance in the processes of respiration. It is considered toxic when it exceeds up to $5 \mathrm{mg} / \mathrm{l}$. According to WHO (1984), its range should be between $0.3 \mathrm{mg} / \mathrm{l}$ in drinking while it varied between 1.94-5.76 $\mathrm{mg} / \mathrm{l}$ in pond. Welch (1952) has also described that most natural waters contain more than $5 \mathrm{mg} / \mathrm{l}$ iron without being toxic due to the buffer action of organic compounds or calcium salts. Javed (1999) reported high concentration of iron causes metallic ion pollution.

Lead (Pb): The mean value of lead was varied from $0.14 \pm 0.12$ (Pond-6) to $4.92 \pm 1.66 \mathrm{mg} / \mathrm{l}$ (Pond-4) Fig -1 . The highest value of $\mathrm{Pb}(7.64 \mathrm{mg} / \mathrm{l})$ was recorded in the month of November at pond-4 which indicates water pollution due to the inclusion of industrial effluents, municipal wastes etc. Abel and Green (1981) reported that different wastes of industries and textiles cause 
serious pollution due to the presence of the heavy metal components like lead. Templeton (1995) has described that the lead entered into the water by wastes of industries. Javed (1999)also reported high concentration of $\mathrm{Pb}$ causes metallic ion pollution.

Conclusion: Present research work reveals significant variations in the concentration of heavy metals due to untreated industrial and sewage wastes added into the pond water. The effluents from these sources have adversely affected the quality of water. Among the study ponds those pond which were situated in industrial area (Pond-3 and 4) contributed significant metals pollution towards the pond ecosystem. In these ponds the concentrations of toxic heavy metals and effluent discharging were found moderately higher than the safe limits for sustainable conservation of freshwater fisheries and aquatic habitats. The findings have important implications for the development of effective watershed management strategies for the control of point and diffuse-source pollution. As well as further study will needed to assess the affect of heavy metals in fish muscle and total pond ecosystem in this region.

Acknowledgement: The authors are highly grateful to Dr. Redanur Rahman, Assistant Professor, Institute of Environmental Science, University of Rajshahi for his laboratory support in analysis of heavy metal components.

\section{References}

Abel PD and Green DWJ 1981. Ecological and toxicological studies on invertebrate fauna of two rivers in the northern pennine orefield. In: Say, P. J. and Whiton, B. A. (eds.). Heavy Metals in Northern England. Environmental Biological Aspects. pp. 22-109.

Bush D, Haesloop U, Scheffel HJ and Schirmer M 1988. Fish and their environment in large European river ecosystems. The River Weser, FRG. Sciences de l'eau.7(1): 75-94.

DoF 2008. Matshaw Sampad Unnayan Avijan 2008 (in Bengali). Department of Fisheries, Ministry of Fisheries and Livestock, Dhaka, Bangladesh. pp. 81-85.

Ehshan MA, Hossain MS, Mazid MA, Mollah MFA, Rahman S and Razzaque A 1997. Limnology of Chanda Beel. Bangladesh J. Fish. Res. 1: 31-40.

Gaigher IG and Krause JB 1989. Growth rate of Mozambique tilapia (Oreochromismossambicus) andsilvercarp (Hypophthalmichthys molitrix). Turkish Biol. 12: 34-39.

Harvey HW 1949. On manganese in sea and freshwater. J. Mar. Biol. Assoc., U.K., 28: 155-164.

Haslam SM 1991. River pollution and ecological perspective. CBS Publishers and Distributors, Delhi, India, 253pp.
Hossain MAR 1998. A Study on Physico-Chemical Parameters of Pond Bottom Sediments with Special Reference to Bioavailable Phosphorus. Bangladesh Journal of Fisheries, 21 (1): 105-110.

IUCN 1991. "Caring for the Earth, a strategy for sustainable living". Published by IUCN, UNEP and WWF, IUCN, Gland, Switzerland. 228 pp.

Javed M 1999. Studies on metal eco-toxicity of river Ravi stretch from Shahdera to Head Baloki. Pak. J. Agri. Sci., 2(3): 10621068.

Javed M 2003. Relationships among water, sediments and plankton for the uptake and accumulation of metals in the river Ravi. Indus J. Plant Sci. 2(4): 326-331.

Jop K 1980. Hydrochemical characteristics and pollution of the Biala Prezemsza catchment basin. Acta. Hydrobiol. 22: 179-190.

Kabir AK, Ali MN and Khondaker M 1997. Study on the Zooplankton from Noakhali North flood plain. Dhaka Univ. $J$. Biol. Sci. 6(1): 31-37.

Kupchella CE and Hyland MC 1993. Environmental Science: Living within the System of Nature. Prentice-Hall International, Inc., Kentucky, USA. pp. 1-579.

Manga N 1983. Heavy metal concentrations in the sediments of the tidal section of the River Lagan. Ir. J. Environ. Sci., 2: 60-64.

McCutcheon SC, Martin JL and Barnwell Jr TO 1992. Water quality. In: Maidment, D. R. (Ed.) Handbook of Hydrology. McGrawHill Book Co. Inc., pp. 11.1-11.73.

Meade JW 1989. Aquaculture Management. New York: Van Nostrand Reinhold. 196pp.

Moore JW and Ramamoorthy S 1984. Organic Chemicals in Natural Waters: Applied Monitoring and Impact Assessment. SpringerVerlag New York Inc. pp.168-191.

Nazneen S 1980. Influence of hydrological factors on the seasonal abundance of phytoplankton in Kinjher Lake. Int. Revue ges. Hydrobiol., 65(2): 269-282.

Saha BK and Hossain A 2002. Saldu Beel Fishery of Tangail. Bangladesh J. Zool. 30(2): 187-194.

Takasusuki J, Araujo MRR and Fernandes MN 2004. Effect of water $\mathrm{pH}$ on copper toxicity in the neotropical fish, Prochilodus scrofa (Prochilodinidae). Bull. Environ. Contam. Toxicol. 72: 1075-1082.

Tariq A, Mohammad J and Mohammad A 1996. Pollution studies of the Indus River, Pakistan, through heavy metal and macronutrient contents of fish, sediment and water. Water Res., 30(6): 1337-1344.

Templeton R 1995. Freshwater Fisheries Management, $2^{\text {nd }}$ ed. Fishing News Books, 242pp.

Trivedi RK and Raj R 1997. Water Pollution. Akashdeep Publishing House, New Delhi. pp. 2-3.

Welch PS 1952. Limnological Methods. McGraw Hill Book Co., Inc. New York, 538pp.

WHO 1984. Guideline for drinking water quality, Recommendations. World Health Organization, Geneva. 1: 130.

World Bank 1991. Bangladesh Fisheries Sector Review; Document of World Bank, Agriculture Operation Division, Asia Country Department.

Manuscript received on 24 November 2012 and revised on 24 December 2012 